\title{
Determination of a macro- to micro-scale progression leading to a magnetized plasma disruption
}

Cite as: Phys. Plasmas 27, 022109 (2020); https://doi.org/10.1063/1.5140348

Submitted: 26 November 2019 . Accepted: 15 January 2020 . Published Online: 12 February 2020

Byonghoon Seo (D), Pakorn Wongwaitayakornkul (D), Magnus A. Haw (D), Ryan S. Marshall (D), Hui Li (D), and Paul M. Bellan (D)

\section{AVS Quantum Science}

A new interdisciplinary home for impactful quantum science research and reviews 


\title{
Determination of a macro- to micro-scale progression leading to a magnetized plasma disruption
}

Cite as: Phys. Plasmas 27, 022109 (2020); doi: 10.1063/1.5140348

Submitted: 26 November $2019 \cdot$ Accepted: 15 January 2020 •

Published Online: 12 February 2020

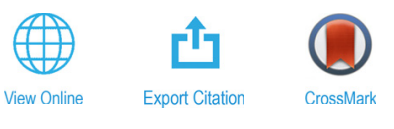

\author{
Byonghoon Seo, ${ }^{1, a)}$ (D) Pakorn Wongwaitayakornkul, ${ }^{1}$ (D) Magnus A. Haw, ${ }^{1, b)}$ (D) Ryan S. Marshall, ${ }^{1}$ (D) Hui Li, ${ }^{2}$ (D \\ and Paul M. Bellan ${ }^{1, C)}$ (iD
}

\author{
AFFILIATIONS \\ ${ }^{7}$ Applied Physics, Caltech, Pasadena, California 91125, USA \\ ${ }^{2}$ Theoretical Division, Los Alamos National Laboratory, Mail Stop B227, Los Alamos, New Mexico 87545, USA \\ ${ }^{a}$ Current address: Department of Physics and Astronomy, UCLA, Los Angeles, CA 90024, USA. \\ Author to whom correspondence should be addressed: bhseo@physics.ucla.edu

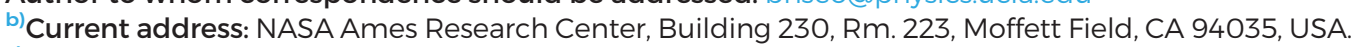 \\ ${ }^{c)}$ Electronic mail: pbellan@caltech.edu
}

\begin{abstract}
We report the observations of a plasma jet evolving through a macro- to micro-scale progression sequence. This leads to a fast magnetic reconnection that results in the jet breaking off from its originating electrode and forming a force-free state. A sausage-like pinching occurs first and squeezes an initially fat, short magnetized jet so that it becomes thin. The thin jet then becomes kink unstable. The lengthening of the jet by the kinking thins the jet even more since the kink is an incompressible instability. When the jet radius becomes comparable to the ion-skin depth, Hall and electron inertial physics become important and establish the environment for a fast magnetic reconnection. This fast reconnection occurs, disrupting the jet and establishing a force-free state. X-ray bursts and whistler waves, evidence of a magnetic reconnection, are observed when the plasma jet breaks off from the electrode. This experimentally observed sequence of successive thinning from pinching followed by kinking is reproduced in a three-dimensional ideal Magnetohydrodynamic (MHD) numerical simulation. The results of the experiment and the numerical simulation, together demonstrate a viable path from macro-scale MHD physics to micro-scale nonMHD physics where fast reconnection occurs.
\end{abstract}

Published under license by AIP Publishing. https://doi.org/10.1063/1.5140348

Magnetohydrodynamic (MHD) current-driven instabilities ${ }^{1-3}$ have long been known to be fundamental to the behavior of magnetically confined plasmas. Magnetic reconnection, another type of instability, is also fundamental because it enables magnetic field topology-changing events such as spheromak formation ${ }^{4,5}$ and solar eruptions. ${ }^{6,7}$ These instabilities are also associated with tokamak stability $^{8,9}$ and impulsive natural phenomena such as solar quasi-periodic pulsations. ${ }^{10,11}$ While finite resistivity enables reconnection in the MHD framework, resistive MHD reconnection is too slow to explain observations. Instead, in most cases of interest, reconnection is governed by much faster microscopic non-MHD processes involving the Hall and electron inertia physics ${ }^{12}$ missing from the more macroscopic MHD description. These Hall and electron inertia effects only become important at spatial scales smaller than the ion skin depth $d_{i}=c / \omega_{p i}$, which is microscopic and not resolved by MHD. However, because of the large scale separation, it is unclear how MHD instabilities can couple to the Hall and electron inertia physics. One possibility is a cascade of MHD instabilities to successively smaller scales until the ion skin depth is reached. ${ }^{13-15}$

Current-driven MHD instabilities are frequently observed in both laboratory and space plasmas ${ }^{3,5,16}$ and are known to be associated with magnetic reconnection. ${ }^{13}$ While it is unclear how the macroscopic current-driven instabilities can couple to microscopic magnetic reconnection, previous theoretical and computational studies have suggested the possibility of a cascade through a transition of successively smaller scale current-driven instabilities. For example, a computational study by Haruki et al. ${ }^{17}$ on current-driven instabilities used a 3D particle-in-cell code to predict the possibility of a sausage-to-kink cascade in the context of high energy particle production by a dense plasma focus. Similarly, a recent analytic study by von der Linden and 
You $^{18}$ suggested a current-driven instability cascade but in an opposite path, i.e., as a kink-to-sausage cascade. These studies suggest that if an MHD cascade results in progressively finer scales, the ion inertia scale and its associated fast non-MHD reconnection might be accessed.

We report here experimental observations showing a sausage-like pinching-to-kink cascade that progresses to the ion inertia scale and then triggers a mechanism that results in fast magnetic reconnection. Here, "fast" reconnection is termed as a Hall-mediated reconnection that could explain an observed magnetic reconnection rate which is much faster than the classical resistive reconnection. The observations show that the sausage-like pinching leads to a kink instability and that because of the inherent incompressibility of the kink instability, the kinking leads to further thinning. This thinning attains the ion inertia scale and so results in fast magnetic reconnection. The observations of a sausage-like pinching to kinking and thinning are reproduced in a 3D numerical MHD simulation.

The experimental configuration ${ }^{5,13,19,20}$ creates an MHD-driven plasma jet which spontaneously develops as a result of magnetic helicity, plasma, and magnetic energy being injected by biased electrodes intercepting poloidal magnetic flux. A cylindrical coordinate system $\{r$, $\theta, z\}$ is used in this paper with the axis defined by the vacuum chamber. The poloidal direction corresponds to $\{r, z\}$, and the toroidal direction is denoted by $\theta$. This electrode setup is topologically identical to that used in spheromak experiments; ${ }^{21-25}$ what is new here is the resolution of the distinct sequence wherein the plasma undergoes sausage-like pinching, then kinks, and then detaches from the originating electrode resulting in a magnetic reconnection and, in a sub-microsecond time scale, the formation of a force-free state (spheromak). The sausage-like pinch to kink sequence followed by the kinking leading to further thinning to attain the ion inertia scale has been reproduced with high space and time resolution in a 3D numerical MHD simulation.

Figure 1 shows the experimental setup. The electrodes consist of a $0.2 \mathrm{~m}$ diameter copper inner disk and a coplanar, concentric $0.5 \mathrm{~m}$ diameter outer copper annulus installed at one end of a $1.4 \mathrm{~m}$ diameter, a $1.6 \mathrm{~m}$ long cylindrical vacuum chamber. ${ }^{20,26} \mathrm{~A}$ bias coil located immediately behind the disk electrode generates an initial dipole-like poloidal magnetic field that links the inner and the outer electrodes and creates the poloidal flux $\psi_{\text {gun }} .{ }^{5}$ The bias coil radius is slightly smaller than the radius of the inner electrode depicted by blue in the top right inset in Fig. 1. The time scale of the bias coil current is several milliseconds, so the linked flux is essentially constant on the experimental time scale which is of the order of microseconds. A controlled amount of hydrogen or nitrogen gas is puffed into the chamber from eight gas nozzles on the disk electrode and eight gas nozzles on the annulus electrode. High voltage from an electronically-switched capacitor bank breaks down this gas in a fraction of a microsecond to form plasma. Eight plasma loops are initially formed where each loop follows the initial dipole poloidal magnetic field (see Ref. 19 for clear images of the eight plasma loops). The inner parts of these loops mutually attract and form a jet which propagates in the $z$ direction away from the electrodes and self-collimates via MHD forces. ${ }^{19,27}$ The amount of injected helicity is controlled by the parameter $\lambda=\mu_{0} I_{\text {gun }} / \psi_{\text {gun }}$, where $I_{\text {gun }}$ is the injected current. Operation regimes are classified based on $\lambda$ as follows: ${ }^{26}$ regime I has low values of $\lambda$ resulting in the formation of a stable, straight plasma jet, regime II has intermediate $\lambda$ involving the formation of a jet that then kinks, ${ }^{5}$ and regime III has high $\lambda$ which is the subject of this paper. The magnetic

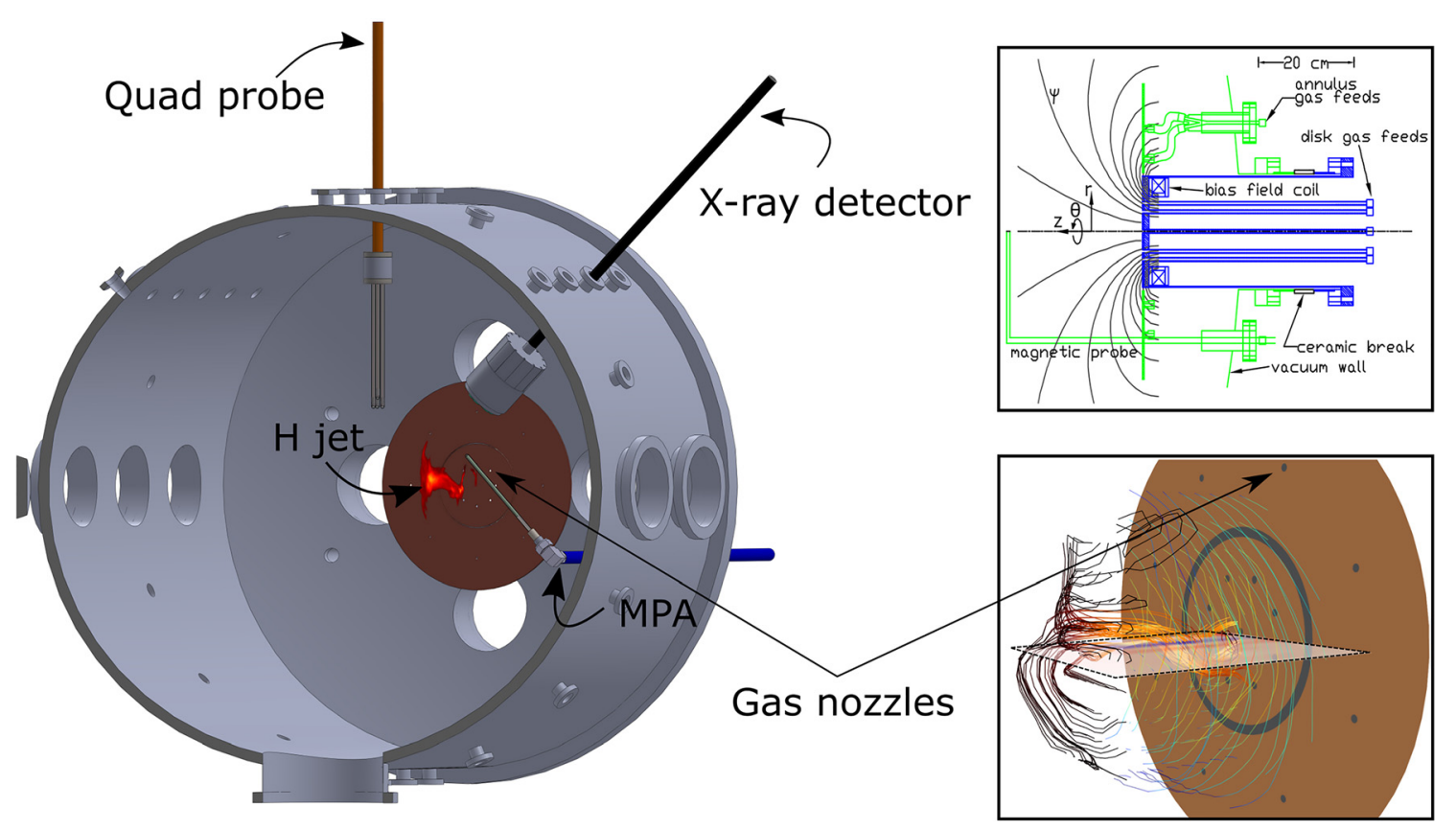

FIG. 1. Experimental setup. The inset at the top right shows a side view of the electrode, the bias coil, and the gas feeds and the inset at the bottom right shows the current and the magnetic field geometry and the 2D plane used for Figs. 4 (b) and 4(c). Red to orange stream lines in the inset indicate currents and blue to green stream lines indicate the magnetic fields. MPA is the magnetic probe array. 
flux in regime III is relatively low at $0.7 \mathrm{mWb}$ and so results in large $\lambda$. The regime III configuration is thus initially rich in toroidal magnetic field energy (i.e., strong poloidal electric current) and so is susceptible to the current-driven MHD instabilities that act to reduce the toroidal magnetic field energy.

Diagnostics include a fast movie camera, a multi-cluster magnetic probe array (MPA), a radio frequency (RF) magnetic probe, and a plastic scintillator X-ray detector, ${ }^{28}$ as shown in Fig. 1 . The MPA is composed of 60 commercial chip inductors that measure at 20 locations, i.e., three inductors at each location provide three orthogonal components of the magnetic field. ${ }^{29}$ Current density is calculated from the measured magnetic field using Ampère's law. The RF magnetic field probe (quad probe in Fig. 1) is composed of four sets of three coils oriented to measure $B_{x}, B_{y}, B_{z}$ with the sets having a tetrahedral structure so that $\nabla \times \mathbf{B}=\mu_{0} \mathbf{J}$ can be determined. The coils are each a single loop so as to provide the fast response time required for measuring high frequency waves. ${ }^{30}$

Most of the data reported here was obtained from hydrogen plasma shots except for the visible-light images in Fig. 2 which were obtained from nitrogen plasma shots. The reason for using these two gases is that as the plasma is radially compressed, its temperature increases and it becomes fully ionized. Since a hydrogen ion is just a proton and so has no bound electrons, a fully ionized hydrogen plasma emits no line radiation, making it difficult to obtain a sharp visible-light image, whereas a nitrogen plasma has a sharp image.

Figure 2 shows a sequence of visible-light images of an evolving nitrogen plasma jet obtained using a fast movie camera. The electrode plane is defined to be $z=0$ (coordinate system shown in $5 \mu$ s frame in Fig. 2). At $1.5 \mu \mathrm{s}$, eight initial plasma-filled arched flux tubes merge to form a single axially moving current-carrying plasma-filled flux tube, i.e., a jet propagating in the $z$ direction (to the left in Fig. 2). At the time of merging, the jet has a large radius $a$ and a small length $L$ so at $1.5 \mu$ s the jet aspect ratio $L / a$ is small. Initial small perturbations grow exponentially during the time interval from 1.5 to $3.0 \mu$ s and pinch the jet so as to reduce $a$ while maintaining $L$ constant; this constitutes a sausage-like instability. This observed pinching is denoted sausage-like because strictly speaking it is not a sausage instability since it does not originate from a perturbation of an initial axisymmetric equilibrium as in the textbook analysis of a sausage instability. However, since MHD force-balance is lost as soon as a textbook sausage instability initiates, the evolution of the observed sausage-like pinching is exactly the same as that of a textbook theoretical sausage instability. Moreover, there is by definition no initial steady state plasma in a coaxial gun configuration powered by a capacitor bank because this configuration necessarily involves a ramping up of a current and so cannot provide an initial steady-state equilibrium. This situation is likely occurring in other experiments having an analogous set-up; ${ }^{21-25}$ i.e., none start from an initial textbook MHD equilibrium. On the other hand, the results of the MHD simulation, which will be described below, show that although the initial condition is set to be the same as the experiment so that there is no initial steady-state equilibrium, a short-lived equilibrium (i.e., force balance) briefly develops just before the onset of the sausage instability.

As a result of the sausage-like pinching decreasing the jet radius $a$, the jet becomes a thin flux rope with increased aspect ratio $L / a$, as seen at $3.5 \mu \mathrm{s}$. The jet becomes kink-unstable at $4 \mu \mathrm{s}$, and the helical deformation caused by the kinking now substantially increases $L$. Moreover, because the kink is an incompressible instability, ${ }^{1}$ the jet volume remains constant during the kink, so this increase in $L$ necessitates a further reduction in $a$ to maintain a constant volume $\sim a^{2} L$. At $4 \mu \mathrm{s}$, the jet length $L$ is approximately three times longer than at $3.5 \mu \mathrm{s}$, and $a$ is reduced by approximately a factor of two. At $4.5 \mu \mathrm{s}$, the jet radius $a$ decreases to be the order of the ion-skin depth; at this time, the jet disrupts and detaches from the electrode, indicating that magnetic reconnection occurs. This disruption is manifested by several distinct simultaneous phenomena, namely, X-ray emission, whistler wave emission, sudden change in the visible-light image indicating that the plasma jet has detached from the electrode, and a change in magnetic topology as indicated by magnetic probes.

The fast magnetic reconnection occurs when $v_{\mathrm{d}} / v_{A} \simeq O(1)$, where $v_{\mathrm{d}}=J / n e$ is the electron drift velocity relative to ions and $v_{A}$ is the Alfvén velocity which is comparable to the ion flow velocity; in this limit, the Hall and electron inertia terms in the electron equation of motion become important. ${ }^{14}$ Using $B_{\theta}=\mu_{0} I / 2 \pi a$ and $B_{z}=\psi /$

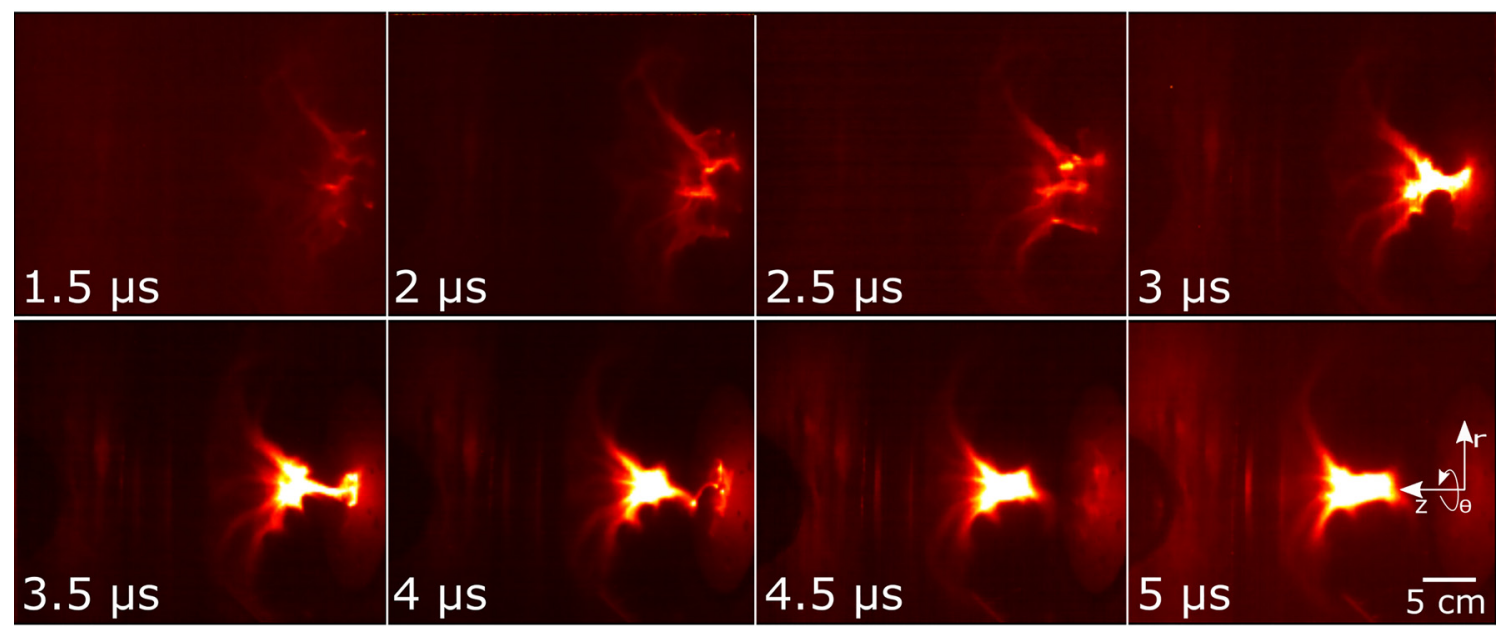

FIG. 2. Time series of a nitrogen plasma shot taken by the fast movie camera. 
$\pi a^{2}$ gives the $v_{d} / v_{A}$ ratio as $v_{d} / v_{A}=\left(I / \pi a^{2} n e\right)\left(\sqrt{\mu_{0} m_{i} n} /\right.$ $\left.\sqrt{\left(\mu_{0} I / 2 \pi a\right)^{2}+\left(\psi / \pi a^{2}\right)^{2}}\right)=\lambda d_{i} / \sqrt{(\lambda a / 2)^{2}+1}$, where $d_{i}$ is the ion skin depth. The numerator $\lambda d_{i} \simeq O(1)$ because $\lambda \sim 100 \mathrm{~m}^{-1}$ and $d_{i} \sim 10^{-2} \mathrm{~m}$. Thus, the regime $a \gg d_{i}$ corresponds to $v_{d} / v_{A} \simeq 0$ and the regime $a \simeq d_{i}$ corresponds to $v_{d} / v_{A} \simeq O(1)$; hence, shrinking of the flux rope radius to $d_{i}$ satisfies the condition for fast magnetic reconnection to occur. Stark-broadening spectroscopy of the hydrogen plasma jet (see Fig. 3) shows that the electron density is $5 \times 10^{21} \mathrm{~m}^{-3}$, which implies a $3 \mathrm{~mm}$ ion skin depth. The observations show that reconnection indeed occurs when the kink self-thinning reduces $a$ to be comparable to the ion skin depth. The resistive skin depth $\sim \sqrt{\eta /\left(\mu_{0} f\right)}$ is about $15 \mathrm{~mm}$ for $T_{e}=2 \mathrm{eV}$ and $4.6 \mathrm{~mm}$ for $T_{e}$ $=10 \mathrm{eV}\left(n_{e}=5 \times 10^{21} \mathrm{~m}^{-3}, \ln \lambda=10\right.$, using $\left.1 / f=0.5 \mu \mathrm{s}\right)$, and so the resistive skin depth is the same order of magnitude as the ion skin depth. As discussed in Ref. 14, finite resistivity will cause the reconnection growth rate to be altered from the collisionless growth rate. Since the resistive skin depth is comparable to $d_{i}$, the magnitude of the reconnection growth rate is thus presumed to differ to some degree from the purely collisionless prediction. Because the thinned kinked flux rope seen at $4.5 \mu \mathrm{s}$ in Fig. 2 is so small (radius less than $3 \mathrm{~mm}$ ), the limited spatial resolution of the available diagnostics could not resolve plasma parameters well enough to make a more definite statement. However, the observation of X-rays and whistler waves discussed below strongly suggests that the whistler-related mechanism underlying collisionless reconnection is operative, but possibly modified by a certain amount of collisionality.

Figure 4(a) shows that current and voltage oscillations, whistler waves, ${ }^{30}$ and X-ray bursts ${ }^{28}$ occur in hydrogen plasma shots at precisely the time the plasma is observed to detach from the electrode. The coincidence of these transient phenomena with plasma detachment supports the presumption that fast magnetic reconnection is associated with the sequence of sausage-like pinching leading to kinking that leads to $a \simeq d_{i}$ when $v_{\mathrm{d}} / v_{A} \simeq O(1)$. The signals in Fig. 4(a)

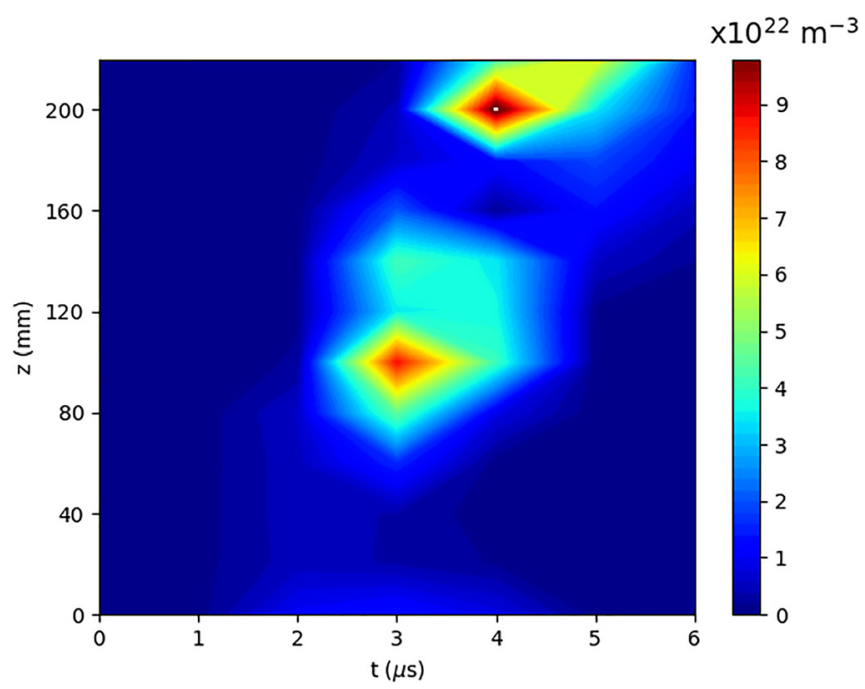

FIG. 3. Position vs time plot of hydrogen density measured by the Stark broadening. at $4 \mu \mathrm{s}$ occur when the jet detaches from the electrode. The $5.5 \mu \mathrm{s} \mathrm{sig-}$ nals are not understood at present but a possible explanation is that the jet detaches from the outer electrode at $5.5 \mu$ s. Figure 4(b) shows the spatial dependence of $\cos \theta=\vec{J} \cdot \vec{B} /(|\vec{J}||\vec{B}|)$ at $5 \mu \mathrm{s}$; this indicates that the current and the magnetic field are nearly parallel in the jet column, so the plasma achieves a nearly force-free state at $5 \mu \mathrm{s} .{ }^{4}$ Figure 4(c) shows the poloidal magnetic field (arrows) and the toroidal magnetic field (colors) at $5 \mu \mathrm{s}$; the existence of closed poloidal magnetic field lines indicates a spheromak-like formation (see Fig. 5 for comparison with the fields at $3.5 \mu$ s, i.e., before the magnetic reconnection occurs). In addition, ion heating was observed at the time when magnetic reconnection happens $(t>4 \mu \mathrm{s})$ and the jet detaches at $z \sim 40 \mathrm{~mm}$, as shown in Fig. 6. This ion heating is also evidence of magnetic reconnection since magnetic reconnection is expected to convert magnetic energy into particle energy.

We have made a 3D ideal MHD numerical simulation of a current-carrying flux rope to model the experimentally observed MHD instability sequence. This simulation, done on the Los Alamos Turquoise cluster, uses part of the Los Alamos COMPutational Astrophysical Simulation Suite (LA-COMPASS). ${ }^{31,32}$ The simulation solves the 3D ideal MHD equations and traces the evolution of mass density $\rho$, pressure $p$, velocity $\vec{v}$, and magnetic field $\vec{B}$ in a numerical Cartesian box of size $2 R_{0}$, where $0.7 R_{0}$ is the initial flux tube radius and $R_{0}=7.14 \mathrm{~cm} .40^{3}$ grid points and a $0.01 t_{A}$ time step were used and the simulation was run from $t=0$ to $t=0.8 t_{A}$, where $t_{A}$ $=2.46 \mu \mathrm{s}$. The center of the Cartesian box is at $x=0, y=0, z=0$. Initial conditions are based on the experimental jet parameters at $2 \mu \mathrm{s}$ and are as follows: (i) a uniform skin current with $I \simeq 90 \mathrm{kA}$ is introduced at $r=5 \mathrm{~cm}$; (ii) the plasma mass density in the flux tube is set to be $\rho_{0}=n m_{H}=5.0 \times 10^{21} \mathrm{u} / \mathrm{m}^{3}$, where $n$ is the density in $\mathrm{m}^{-3}$ and $m_{H}$ is the hydrogen mass in $\mathrm{u}$ and the temperature is $2 \mathrm{eV}$; (iii) a $z$-directed magnetic field $B_{z \text {,sim }}=224 \mathrm{G}$ is uniformly applied in the simulation domain; (iv) small perturbations are added to the density to induce the instabilities. The total initial density is $\rho(\mathbf{r})=\rho_{\text {main }}$ $+\rho_{\text {sausage }}+\rho_{\text {kink }}$, where the main loop density and the respective perturbations for the kink and sausage modes are $\rho_{\text {main }}=\rho_{0} \exp \left(-r^{2} /\right.$ $\left.2 \sigma_{0}^{2}\right), \rho_{\text {sausage }}=0.1 \rho_{0} \exp \left(-r^{2} / 2\left(\sigma_{0}-\sigma_{s} \cos \left(k_{\text {sausage }} z\right)\right)^{2}\right)$, and $\rho_{\text {kink }}$ $=0.1 \rho_{0} \exp \left(-\left(\mathbf{r}-\mathbf{r}^{\prime}\right)^{2} / R_{0}^{2}\right) \exp \left(-r^{2} / 2 \sigma_{k}^{2}\right)$. Here, $\mathbf{r}^{\prime}=r_{0}\left[\cos \left(k_{\text {kink }} z\right) \hat{x}\right.$ $\left.+\sin \left(k_{\text {kink }} z\right) \hat{y}\right], \sigma_{0}=3.6 \mathrm{~cm}, \sigma_{s}=0.36 \mathrm{~cm}, \sigma_{k}=2.2 \mathrm{~cm}$, and $r_{0}$ $=0.7 \mathrm{~cm}$. Respective wavenumbers $k_{\text {kink }}=1.4 \times 10^{-2} \mathrm{~m}^{-1}$ and $k_{\text {sausage }}$ $=1.3 \times 10^{-2} \mathrm{~m}^{-1}$ are chosen corresponding to the unstable modes observed in the experiment. A small flow velocity, $6 \mathrm{~km} / \mathrm{s}$ at the top and bottom and linearly decreasing on approaching the center $(z=0)$, is initially imposed in the $\pm z$ directions to simulate the axial motion of the jet. This flow velocity does not play a major role but provides an imbalance between the periodic bulged structures to mimic the axial motion of the pressure gradient in $\pm z$ direction.

Figure 7 shows the simulation results as a time sequence of the current density iso-surfaces. A flux tube evolves with an initial skin current configuration and an initial small aspect ratio $L / a$. The initial condition was set to be the same as the experiment, so the flux tube is initially not in equilibrium and the inward radial force pinches the flux tube. Since the magnetic and the thermal pressures increase by the radial pinching, a radial force balance is established at $2.7 \mu$ s and the flux tube becomes stable (see Fig. 8). Then, the flux tube develops a sausage instability at $3.3 \mu \mathrm{s}$. The sausaging increases $L / a$ to form a highly collimated current channel. A kink instability spontaneously starts at $3.6 \mu$ s. The numerical 

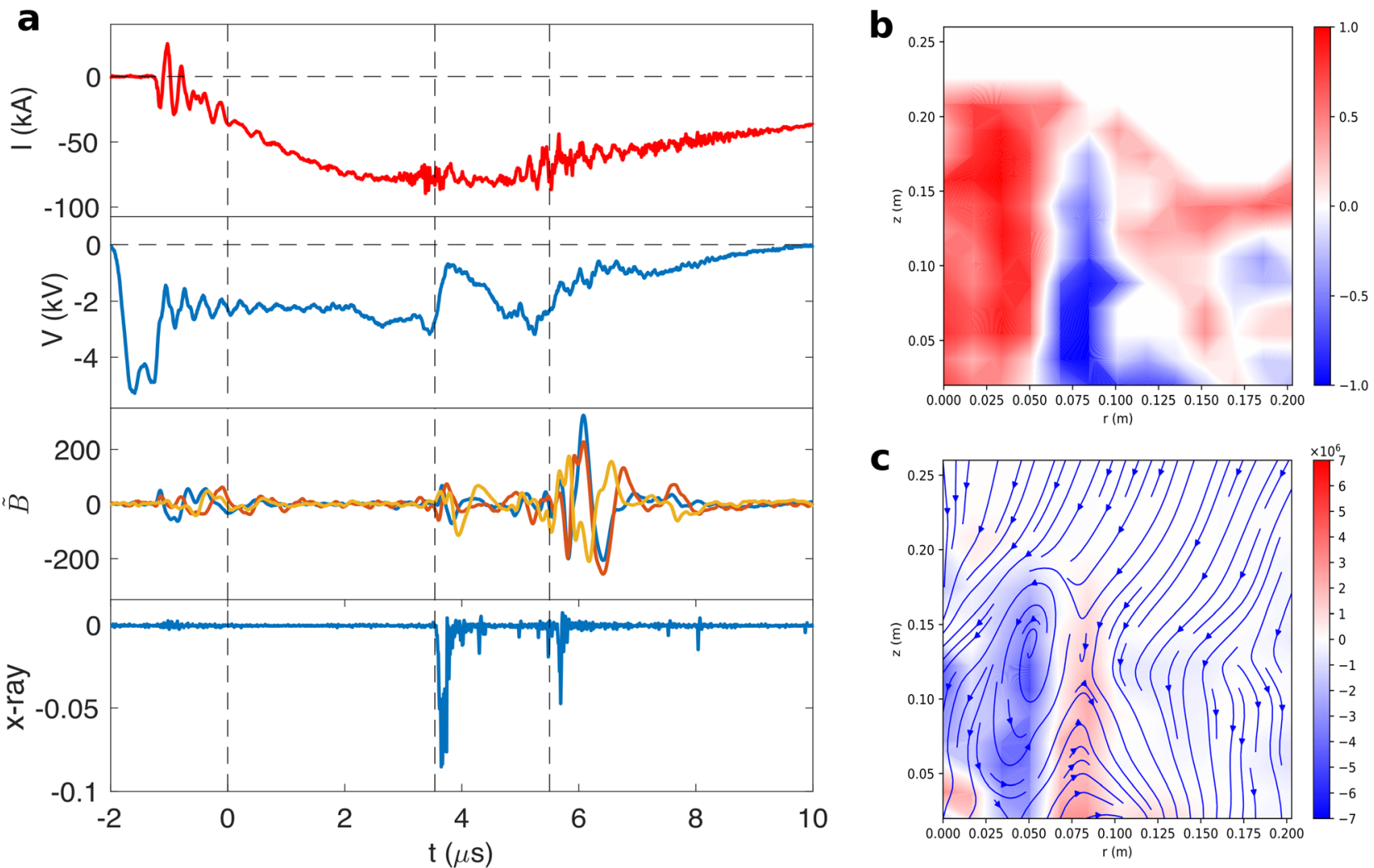

FIG. 4. (a) (From top) Time dependent current, voltage, $\tilde{\mathbf{B}}$ (waves) measured by the RF magnetic wave probe, and X-ray bursts obtained from hydrogen plasma shots. A bandpass filter was applied to $\tilde{\mathbf{B}}$ in the frequency range of $1-5 \mathrm{MHz}$ which is in the whistler wave regime. (b) Spatial dependence of $\cos \theta=\vec{J} \cdot \vec{B} /(|\vec{J}||\vec{B}|)$ at $5 \mu$ s indicating a near force-free state (c) poloidal magnetic fields (streamlines) and $J_{\theta}$ (contour) at $5 \mu$ s. The MPA was used to obtain (b) and (c).

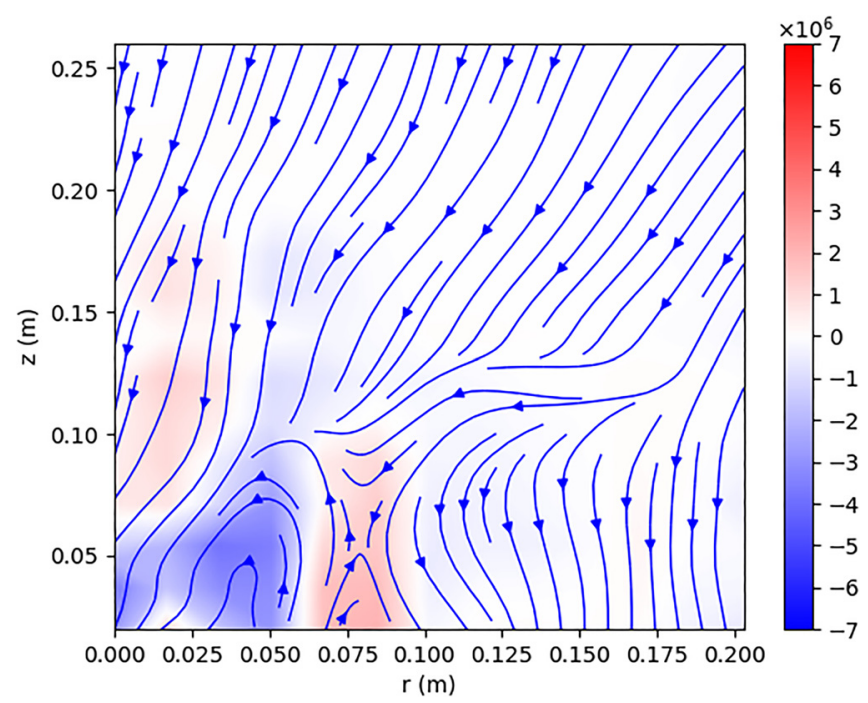

FIG. 5. Poloidal field (streamlines) and toroidal current density (color contour) at $3.5 \mu \mathrm{s}$ which is before magnetic reconnection occurs. simulation thus confirms that the flux tube can transition from being sausage unstable to being kink unstable.

In addition, the simulation shows that regions where $v_{d} / v_{A}$ $\sim O(1)$ develop when the jet kinks implying that non-ideal MHD physics and resulting fast reconnection should occur at these locations (see Fig. 9). Being MHD, the simulation does not replicate the fast non-MHD physics, but simply indicates when and where the MHD physics has accessed the ion skin depth scale.

In conclusion, we have experimentally observed a plasma jet transition from a sausage-like pinching to a kink instability which then leads to magnetic reconnection. The sausage-like pinching occurs first and pinches a fat, short magnetized jet to become a thin, long magnetized jet. The thin, long jet then becomes kink unstable. The incompressible kinked jet further lengthens and, to maintain a constant volume, the kinked jet thins even more. Because of this thinning, the jet radius becomes comparable to the ion-skin depth setting off Hall and electron inertial physics that result in fast magnetic reconnection, jet disruption, and the establishment of a nearly force-free state. The observation of Xray bursts and whistler waves, evidence of fast magnetic reconnection, validate that non-MHD physics has been accessed. The experimentally observed progression to ion-skin depth scales has been confirmed by a three-dimensional ideal MHD numerical simulation. The experiment and its numerical confirmation together establish a mechanism linking macroscale ideal MHD (no reconnection, scale exceeding ion skin 

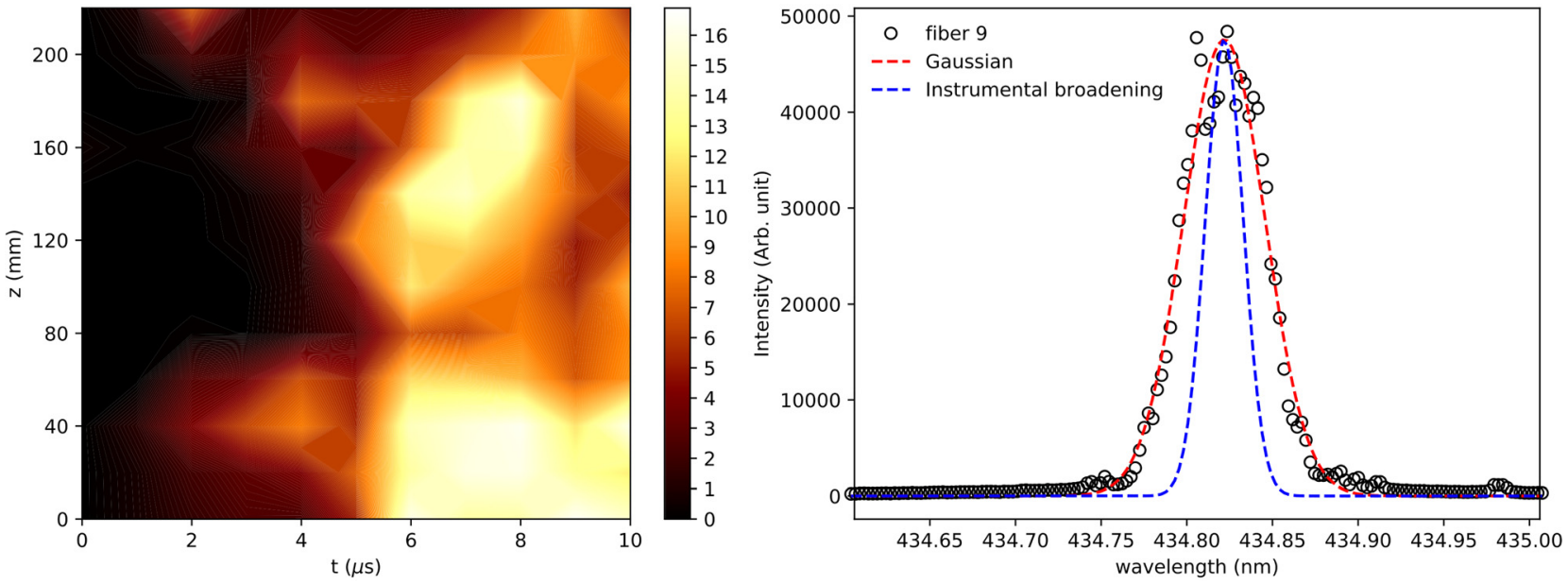

FIG. 6. (Left) Position vs time plot of ion temperature obtained from nitrogen ion Doppler broadening measurements at $434.8 \mathrm{~nm}$; colorbar is in eV. (Right) Sample Doppler broadened spectrum.
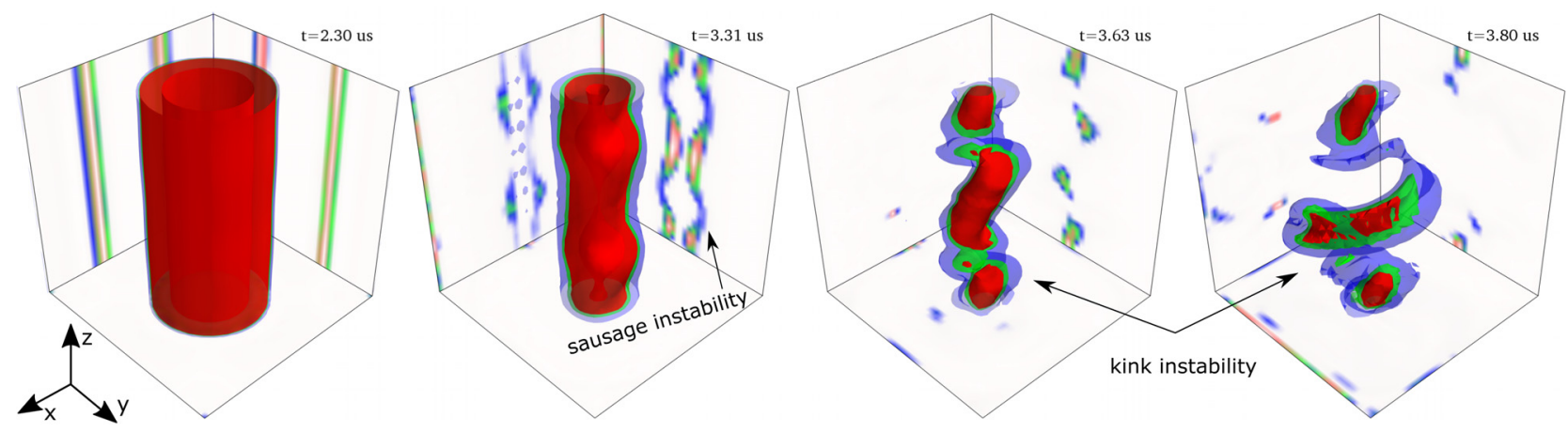

FIG. 7. Time sequence of the current density iso-surfaces obtained from simulation \# 471 . The two back planes at each time, respectively, show the cross sections of the current density at $x=0$ and at $y=0$. The surface plots contain three iso-surfaces at levels 0.19 (blue), 0.32 (green), and 0.41 (red) of the maximum current density at each time.

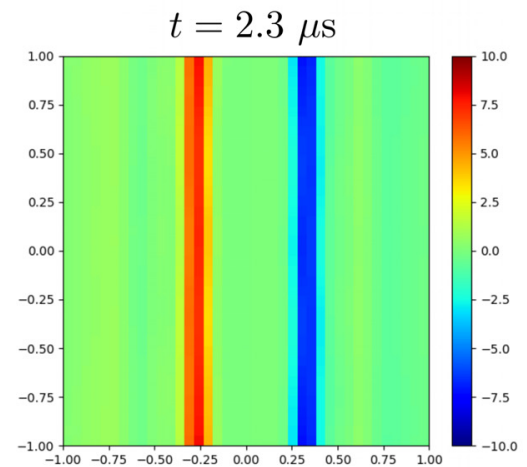

Foce imbalance

$$
F_{y}=(\vec{J} \times \vec{B}-\vec{\nabla} P) \cdot \hat{y}
$$

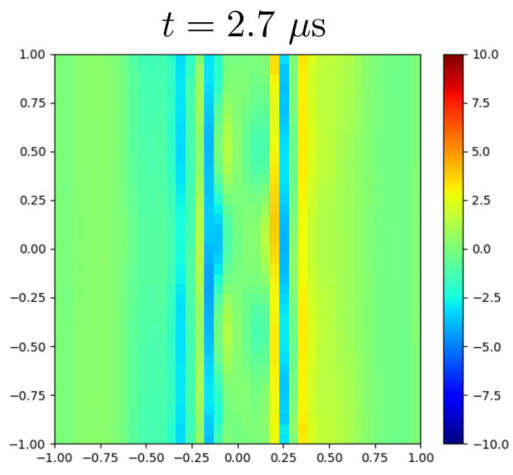

Foce balance

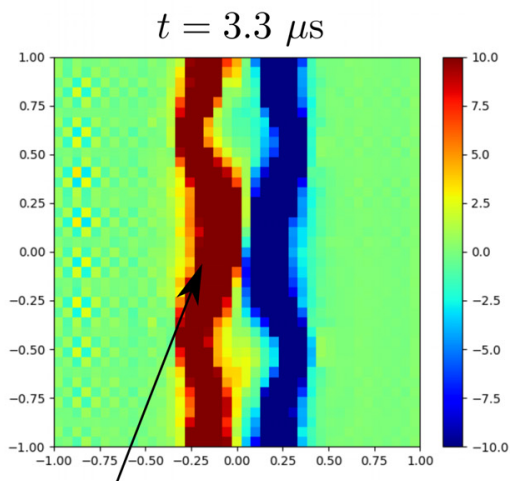

Sausage instability

FIG. 8. Time-dependent radial force obtained from the numerical simulation. Initially, the flux tube is not in equilibrium so the direction of the radial force at $2.3 \mu$ s is inward. By pinching the flux tube, a temporary radial force balance is established at $2.7 \mu \mathrm{s}$. Sausage instability occurs at $3.3 \mu \mathrm{s}$. 


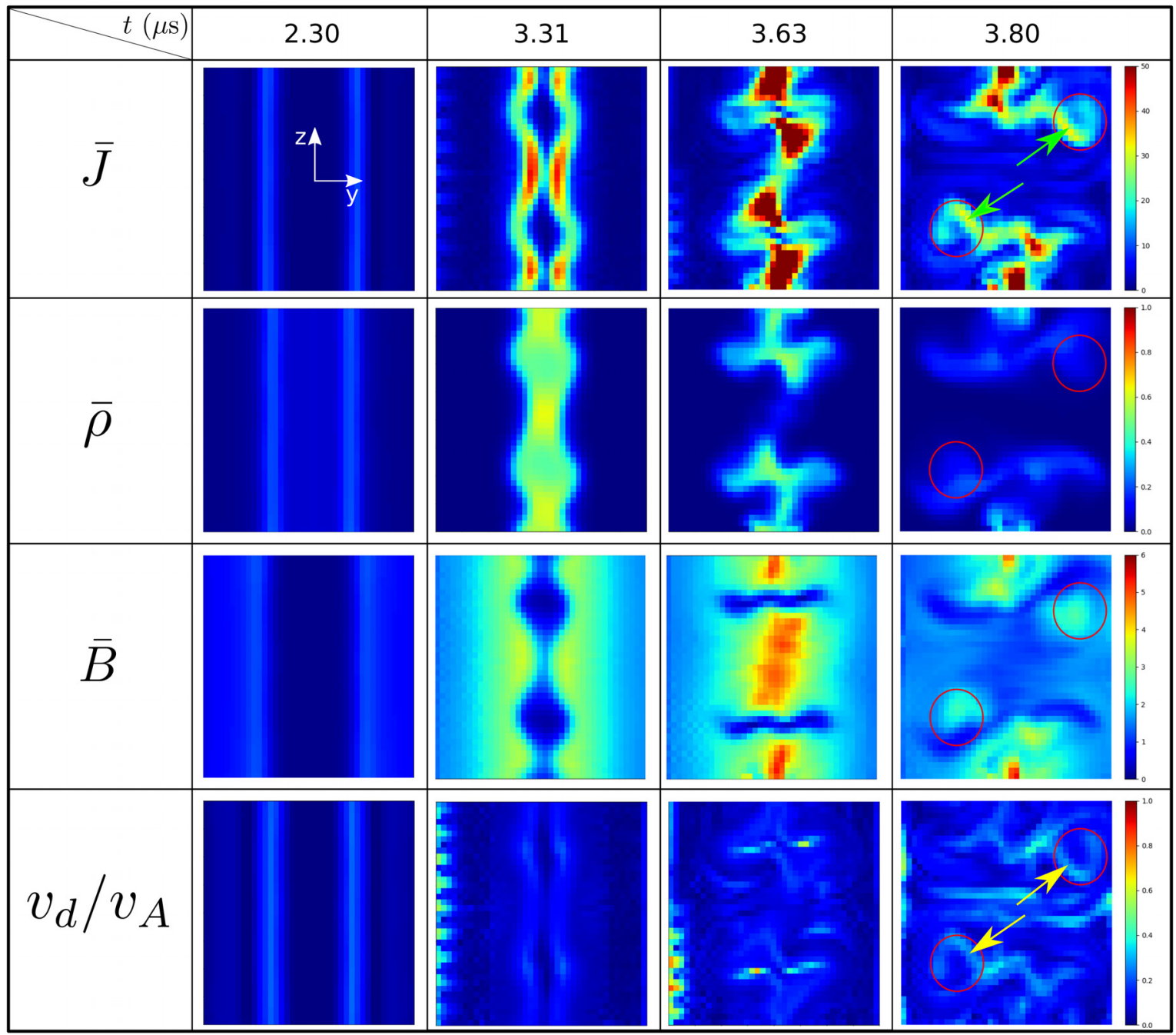

FIG. 9. Time sequence of $\bar{J}, \bar{\rho}, \bar{B}$, and $v_{d} / v_{A}$ in the $y-z(x=0)$ plane obtained from simulation \# 471 . The $\bar{J}$ plots are the same as the back right planes in Fig. 5 but are shown with continuous color contours. The flux rope becomes sausage unstable at $3.31 \mu \mathrm{s}$ and then kink unstable so the flux rope kinks into and out of the page after $3.63 \mu$ $\mathrm{s}$. The regions where the flux rope passes through this plane are indicated by red circles. The ratio $v_{d} / v_{A}$ becomes large at the locations indicated by yellow arrows at $3.80 \mu$ $s$ because $\bar{J}$ becomes large at these locations as indicated by green arrows.

depth) to microscale physics (fast reconnection, scale less than ion skin depth, X-rays, whistler waves).

This material is based upon the work supported by the NSF/DOE Partnership in Plasma Science via the U.S. Department of Energy Office of Science, Office of Fusion Energy Sciences Award No. DEFG02-04ER54755, by the USDOE ARPA-E Grant via Award No. DEAR0000565, by the NSF Division of Atmospheric and Geospace Sciences via Award No. 1914599, and by the Air Force Office of Scientific Research via Award No. FA9550-11-1-0184.

\section{REFERENCES}

${ }^{1}$ W. A. Newcomb, Ann. Phys. 10, 232 (1960).

${ }^{2}$ T. D. Arber and D. F. Howell, Phys. Plasmas 3, 554 (1996).
${ }^{3}$ U. Shumlak, R. P. Golingo, B. A. Nelson, and D. J. Den Hartog, Phys. Rev Lett. 87, 205005 (2001)..

${ }^{4}$ J. B. Taylor, Rev. Mod. Phys. 58, 741 (1986).

${ }^{5}$ S. C. Hsu and P. M. Bellan, Phys. Rev. Lett. 90, 215002 (2003).

${ }^{6}$ K. Shibata, Y. Ishido, L. W. Acton, K. T. Strong, T. Hirayama, Y. Uchida, A. H. McAllister, R. Matsumoto, S. Tsuneta, T. Shimizu et al., Publ. Astron. Soc. Jpn. 44, L173 (1992).

${ }^{7}$ A. C. Sterling, R. L. Moore, D. A. Falconer, and M. Adams, Nature 523, 437 (2015). ${ }^{8}$ MN. Rosenbluth, Phys. Fluids 16, 1894 (1973).

${ }^{9}$ K. L. Wong, M. S. Chu, T. C. Luce, C. C. Petty, P. A. Politzer, R. Prater, L. Chen, R. W. Harvey, M. E. Austin, L. C. Johnson et al., Phys. Rev. Lett. 85, 996 (2000).

${ }^{10}$ H. Tian, P. R. Young, K. K. Reeves, T. Wang, P. Antolin, B. Chen, and J. He, Astrophys. J. 823, L16 (2016).

${ }^{11}$ B. R. Dennis, A. K. Tolbert, A. Inglis, J. Ireland, T. Wang, G. D. Holman, L. A. Hayes, and P. T. Gallagher, Astrophys. J. 836, 84 (2017)..

${ }^{12}$ Y. Ren, M. Yamada, S. Gerhardt, H. Ji, R. Kulsrud, and A. Kuritsyn, Phys. Rev. Lett. 95, 055003 (2005). 
${ }^{13}$ A. L. Moser and P. M. Bellan, Nature 482, 379 (2012).

${ }^{14}$ P. M. Bellan, Phys. Plasmas 21, 102108 (2014).

${ }^{15}$ X. Zhai and P. M. Bellan, Phys. Plasmas 23, 032121 (2016)..

${ }^{16}$ D. M. Rust and B. J. LaBonte, Astrophys. J. 622, L69 (2005).

${ }^{17}$ T. Haruki, H. R. Yousefi, K. Masugata, J. I. Sakai, Y. Mizuguchi, N. Makino, and H. Ito, Phys. Plasmas 13, 082106 (2006).

${ }^{18} \mathrm{~J}$. Von der Linden and S. You, Phys. Plasmas 24, 052105 (2017).

${ }^{19}$ S. You, G. S. Yun, and P. M. Bellan, Phys. Rev. Lett. 95, 045002 (2005).

${ }^{20}$ B. Seo and P. M. Bellan, Phys. Plasmas 25, 112703 (2018)..

${ }^{21}$ T. R. Jarboe, I. Henins, A. R. Sherwood, C. W. Barnes, and H. W. Hoida, Phys. Rev. Lett. 51, 39 (1983).

${ }^{22}$ H. S. McLean, S. Woodruff, E. B. Hooper, R. H. Bulmer, D. N. Hill, C. Holcomb, J. Moller, B. W. Stallard, R. D. Wood, and Z. Wang, Phys. Rev. Lett. 88, 125004 (2002)..

${ }^{23}$ C. D. Cothran, A. Falk, A. Fefferman, M. Landreman, M. R. Brown, and M. J. Schaffer, Phys. Plasmas 10, 1748 (2003)..
${ }^{24}$ A. al-Karkhy, P. K. Browning, G. Cunningham, S. J. Gee, and M. G. Rusbridge, Phys. Rev. Lett. 70, 1814 (1993)..

${ }^{25}$ M. Nagata, T. Kanki, T. Masuda, S. Naito, H. Tatsumi, and T. Uyama, Phys. Rev. Lett. 71, 4342 (1993)..

${ }^{26}$ S. C. Hsu and P. M. Bellan, Phys. Plasmas 12, 032103 (2005).

${ }^{27}$ P. M. Bellan, Phys. Plasmas 10, 1999 (2003).

${ }^{28}$ S. Marshall, M. J. Flynn, and P. M. Bellan, Phys. Plasmas 25, 112101 (2018).

${ }^{29}$ C. A. Romero-Talamas, P. M. Bellan, and S. C. Hsu, Rev. Sci. Instrum. 75, 2664 (2004).

${ }^{30}$ M. A. Haw, B. Seo, and P. M. Bellan, Geophys. Res. Lett. 46, 7105-7112, https://doi.org/10.1029/2019GL082621 (2019).

${ }^{31}$ H. L. S. Li, Technical Report No. LA-UR-03-8926, Los Alamos National Lab, 2003.

${ }^{32}$ M. A. Haw, P. Wongwaitayakornkul, H. Li, and P. M. Bellan, Astrophys. J. 862, L15 (2018). 